Tandem Conversion of Fructose to 2, 5-Dimethylfuran with the Aid of

\title{
Ionic Liquids
}

Changhui Zhu ${ }^{\text {a, b, c, d, Haiyong Wang a, b,c, d, Chiliu Cai }}{ }^{\text {a, b, c }}$, Kang Bi ${ }^{\text {f }}$, Bijuan

Cai e, Xiangbo Song a, b, c, d, Qiying Liu a, b, c, g*, Longlong Ma ${ }^{\text {a, b, c }}$

a Guangzhou Institute of Energy Conversion, Chinese Academy of Sciences, Guangzhou 510640, P. R. China

b Key Laboratory of Renewable Energy, Chinese Academy of Sciences, Guangzhou 510640, P. R. China

c Guangdong Key Laboratory of New and Renewable Energy Research and Development, Guangzhou 510640, P. R. China

${ }^{\mathrm{d}}$ University of Chinese Academy of Sciences, Beijing 100049, P. R. China

e College of Materials and Energy, South China Agricultural University, Guangzhou 510642, P. R. China

${ }^{\mathrm{f}}$ Shandong Province Metallurgical Engineering Company Limited, Jinan 250101, P. R. China

${ }^{g}$ Dalian National Laboratory for Clean Energy, University of Chinese Academy of Sciences, Dalian 116023, P. R. China

* Corresponding author:

Qiying Liu, Tel: +86 20 37029835; Fax: +86 20 87057789, E-mail: liuqy@ms.giec.ac.cn, Address: No. 2, Wushan Nengyuan Road, Tianhe District, Guangzhou, P. R. China 
Supporting Information includes:

Figure S1. GC-distribution of the products.

Figure S2. GC-distribution of small molecular alkanes in exhaust gas.

Figure S3. HPLC-distribution of reaction solution collected.

Table S1. Main intermediates yielded from fructose via tandem reaction in Figure 5.

Table S2. Main intermediates yielded from fructose via tandem reaction in Figure 6.

Figure S4. Continue production of HMF and DMF from fructose via tandem reaction without dehydration catalyst.

Table S3. Main intermediates yield from fructose via tandem reaction over $\mathrm{NbOPO}_{4}$ in Figure 7.

Table S4. Main intermediates yield from fructose via tandem reaction with different [BMim]Cl concentration in Figure 8.

Scheme S1. Proposed interaction mechanism between (A) fructose and [BMim] $]^{+}$, and interaction mechanism between (B) HMF and [BMim $]^{+}$.

Figure S5. FTIR spectra of (A) fructose, (B) fructose in [BMim]Cl, (C) [BMim]Cl, (D) $\mathrm{HMF}$, and (E) HMF in [BMim]Cl.

Figure S6. $\mathrm{NH}_{3}$-TPD profiles obtained on (A) $\mathrm{HY}$ zeolite and (B) $\mathrm{NbOPO}_{4}$.

Figure S7. XRD patterns for the fresh and spent catalyst of (A) HY zeolite, (B) $\mathrm{NbOPO}_{4}$, and (C) $\mathrm{Cu}-\mathrm{Ru} / \mathrm{C}$.

Figure S8. Average particle size distribution of $\mathrm{Cu}$, $\mathrm{Ru}$ over (A) fresh $\mathrm{Cu}-\mathrm{Ru} / \mathrm{C}$ and (B) spent $\mathrm{Cu}-\mathrm{Ru} / \mathrm{C}$. 


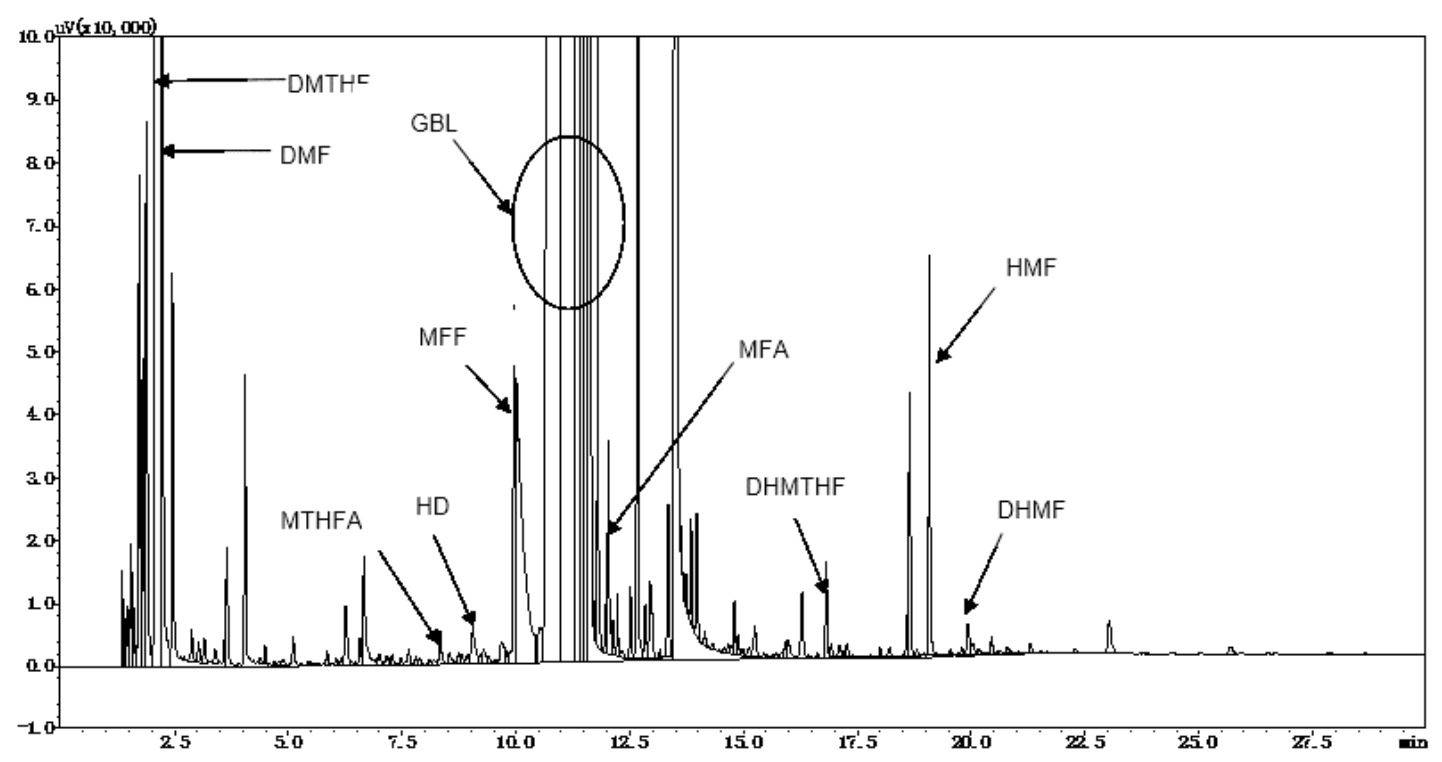

Figure S1. GC-distribution of the products.

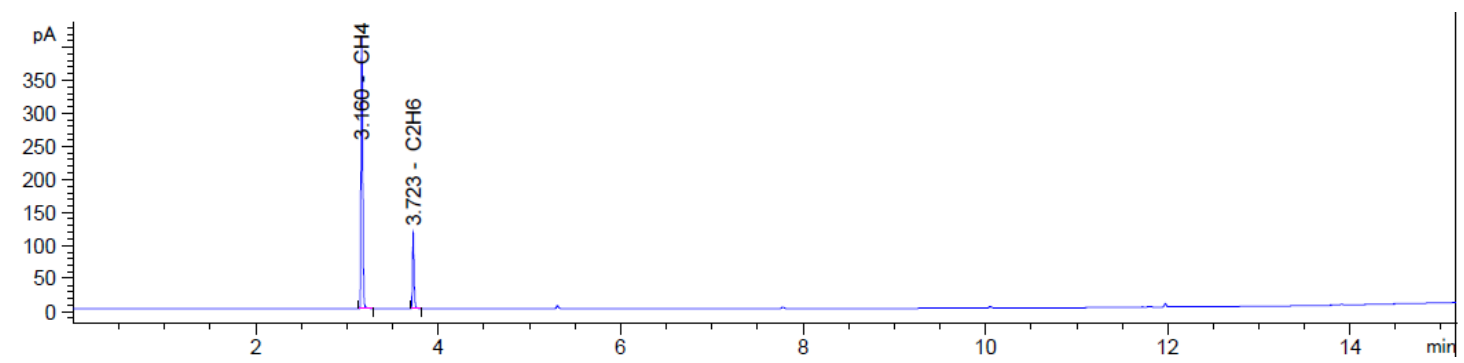

Figure S2. GC-distribution of small molecular alkanes in exhaust gas.

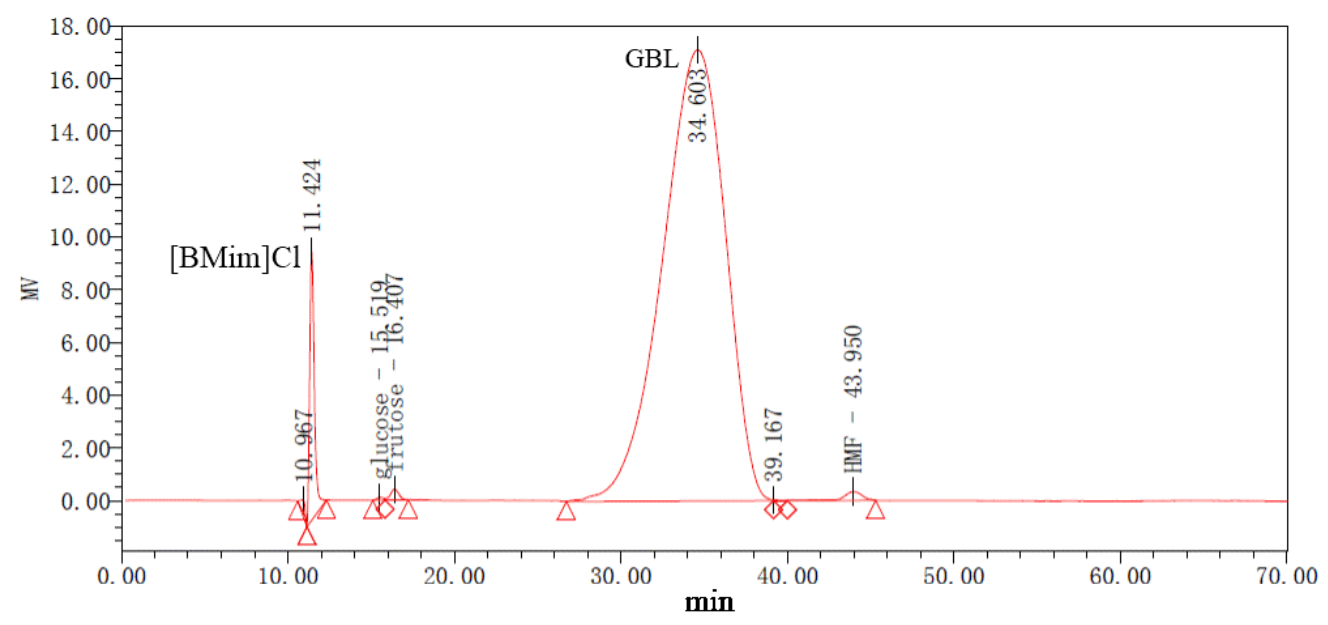

Figure S3. HPLC-distribution of reaction solution collected. 


\begin{tabular}{lll}
\hline $\mathrm{t}(\mathrm{h})$ & DHMF (\%) & MFF (\%) \\
\hline 12 & 1.3 & 0.4 \\
24 & 2.7 & 0.9 \\
36 & 3.3 & 0.8 \\
48 & 0.6 & 0.3 \\
60 & 0.9 & 0.3 \\
\hline
\end{tabular}

Table S1. Main intermediates yielded from fructose via tandem reaction in Figure 5.

\begin{tabular}{lll}
\hline $\mathrm{t}(\mathrm{h})$ & DHMF (\%) & MFF (\%) \\
\hline 12 & 3.3 & 1.4 \\
24 & 4.1 & 1.3 \\
36 & 2.3 & 1.1 \\
48 & 1.7 & 0.9 \\
60 & 1.6 & 0.9 \\
\hline
\end{tabular}

Table S2. Main intermediates yielded from fructose via tandem reaction in Figure 6.

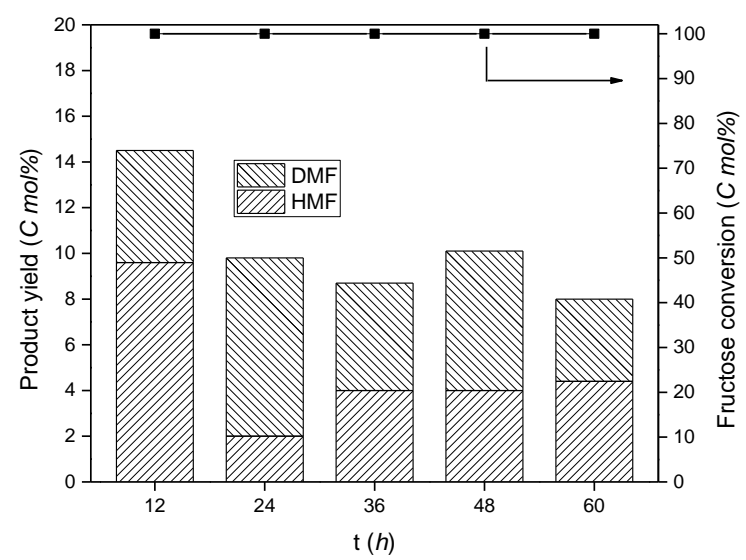

Figure S4. Continue production of HMF and DMF from fructose via tandem reaction without dehydration catalyst.

Reaction condition: Reactor I : $\mathrm{SiO}_{2}=1 \mathrm{~g}$, substrate fructose $=1 \mathrm{wt} . \%$, liquid flow rate $=0.01 \mathrm{~mL} \cdot \mathrm{min}^{-1}, \mathrm{WHSV}_{\text {(fructose) }}=0.007 \mathrm{~h}^{-1}, \mathrm{~T}=120^{\circ} \mathrm{C} ;$ Reactor II $: \mathrm{Cu}-\mathrm{Ru} / \mathrm{C}=1 \mathrm{~g}, \mathrm{H}_{2}$ flow rate $=30 \mathrm{~mL} \cdot \mathrm{min}^{-1}, \mathrm{H}_{2}$ pressure $=5 \mathrm{MPa}, \mathrm{T}=180^{\circ} \mathrm{C}$. 


\begin{tabular}{lll}
\hline $\mathrm{t}(\mathrm{h})$ & DHMF (\%) & MFF (\%) \\
\hline 12 & 9.6 & 1.3 \\
24 & 7.1 & 0.9 \\
36 & 3.6 & 0.3 \\
48 & 2.4 & - \\
60 & 3.3 & 0.7 \\
\hline
\end{tabular}

Table S3. Main intermediates yield from fructose via tandem reaction over $\mathrm{NbOPO}_{4}$ in Figure 7.

(A) $10 w t . \% \mathrm{H}_{2} \mathrm{O}$

\begin{tabular}{lll}
\hline $\mathrm{t}(\mathrm{h})$ & $\mathrm{DHMF}(\%)$ & $\mathrm{MFF}(\%)$ \\
\hline 12 & 0.5 & 0.1 \\
24 & 0.6 & 0.1 \\
36 & 1.3 & 0.3 \\
48 & 1 & 0.2 \\
60 & 0.6 & - \\
\hline
\end{tabular}

(B) $5 w t . \%[\mathrm{BMim}] \mathrm{Cl}$

\begin{tabular}{lll}
\hline $\mathrm{t}(\mathrm{h})$ & $\mathrm{DHMF}(\%)$ & $\mathrm{MFF}(\%)$ \\
\hline 12 & 2.3 & 0.5 \\
24 & 3.7 & 0.6 \\
36 & 2.3 & 0.3 \\
48 & 1.7 & - \\
60 & 1.6 & 0.1 \\
\hline
\end{tabular}


(C) $15 w t . \%[\mathrm{BMim}] \mathrm{Cl}$

\begin{tabular}{lll}
\hline $\mathrm{t}(\mathrm{h})$ & DHMF (\%) & MFF (\%) \\
\hline 12 & 0.6 & 0.5 \\
24 & 2.7 & 0.5 \\
36 & 3.3 & 0.3 \\
48 & 1.9 & 0.3 \\
60 & 0.6 & 0.1 \\
\hline
\end{tabular}

Table S4. Main intermediates yield from fructose via tandem reaction with different [BMim] $\mathrm{Cl}$ concentration in Figure 8.

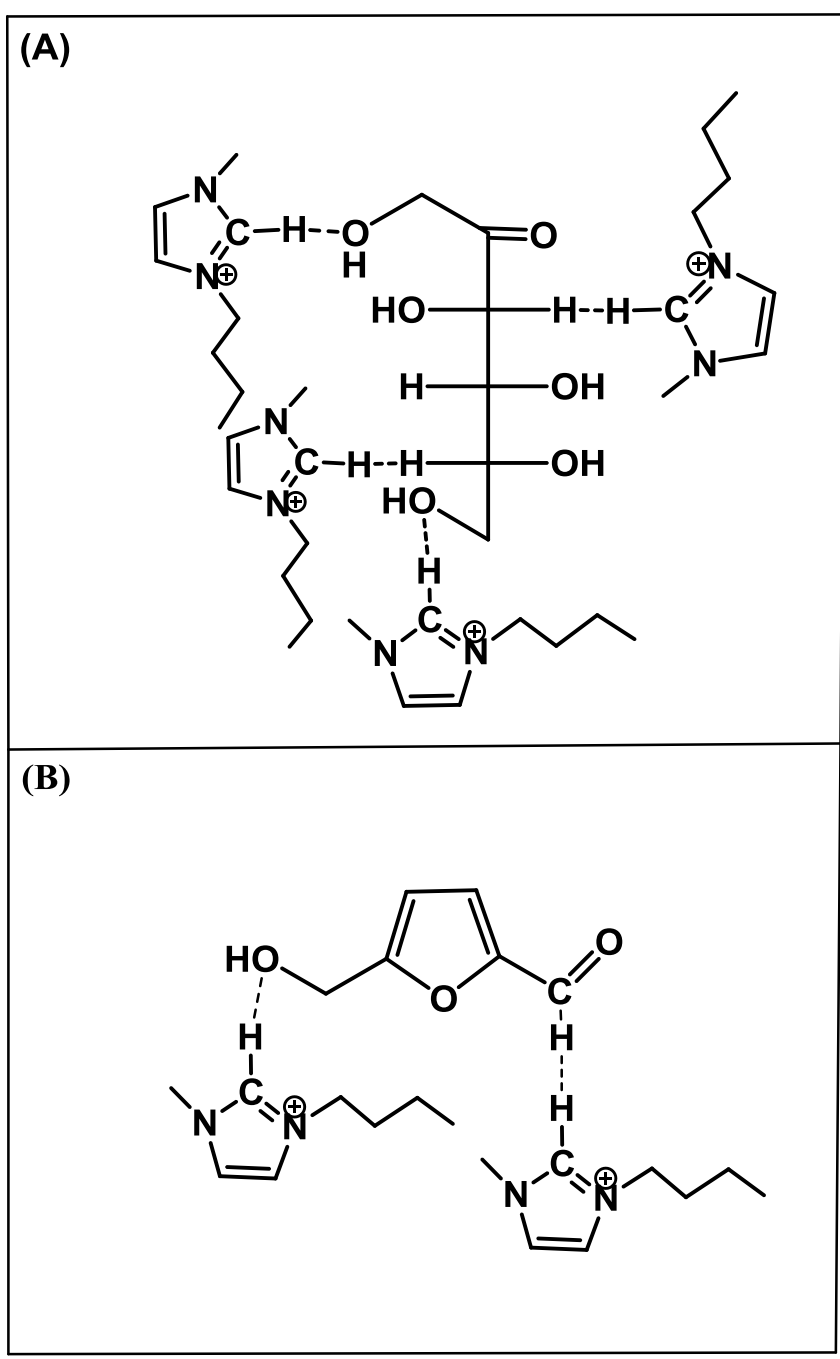

Scheme S1. Proposed interaction mechanism of (A) fructose and $[\mathrm{BMim}]^{+}$, and (B) HMF and [BMim $]^{+}$. 
We conducted the liquid FTIR to confirm the effect of [BMim]Cl on fructose and $\mathrm{HMF}$, with the analysis verified in Figure S5. As to the effect of [BMim]Cl on fructose, the absorption peaks at $3530 \mathrm{~cm}^{-1}$ assigned to the stretching vibration of $-\mathrm{OH}$ and at $3014 \mathrm{~cm}^{-1}$ representing asymmetric vibration of $\mathrm{CH}_{2}$ (from $\mathrm{C}_{6}$ ) in fructose ${ }^{1}$ were broaden in [BMim]Cl system by comparison Figure S5A and Figure S5B. In addition, absorption peaks at $2980 \mathrm{~cm}^{-1}$, and $2900 \mathrm{~cm}^{-1}$ assigned to stretching vibration of $\mathrm{CH}_{2}$ of $\mathrm{C}_{1}$, along with and $875 \mathrm{~cm}^{-1}$ belonged to stretching vibration of $\mathrm{C}-\mathrm{C}+$ bending vibration of $\mathrm{C}-\mathrm{CH}+$ bending vibration of $\mathrm{CH}$ from pyranose ring in fructose ${ }^{1}$ were also broaden, thus indicating the presence of hydrogen bond between $-\mathrm{OH}$ and $\mathrm{Cl}^{-}$. When it referred the effect of [BMim]Cl on $\mathrm{HMF}$, the absorption peak at around $1200 \mathrm{~cm}^{-1}$ in the pure HMF is attributed to aldehyde $\mathrm{C}-\mathrm{H}$ bending ${ }^{1}$, whereas absorption peak of aldehyde $\mathrm{C}-\mathrm{H}$ of $\mathrm{HMF}$ in $[\mathrm{BMim}] \mathrm{Cl}$ was broaden into $\mathrm{C}-\mathrm{N}$ stretching peak from $[\mathrm{BMim}] \mathrm{Cl}^{2}$.

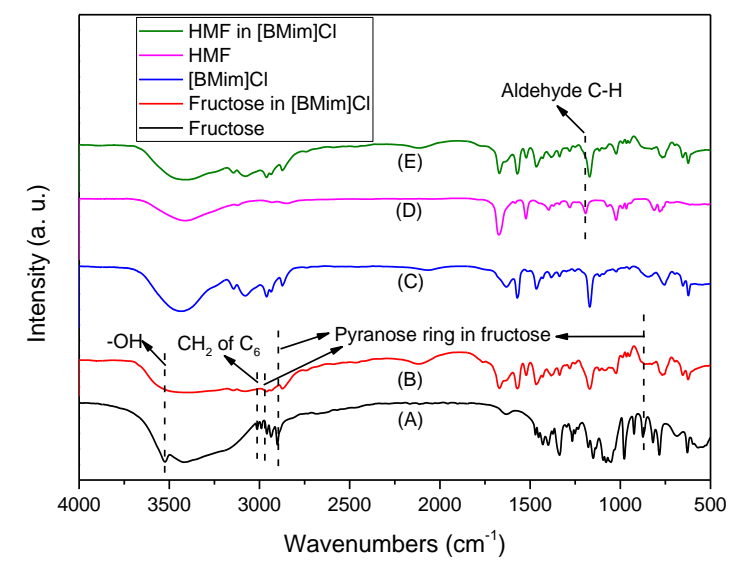

Figure S5. FTIR spectra of (A) fructose, (B) fructose in [BMim]Cl, (C) [BMim]Cl, (D) $\mathrm{HMF}$, and (E) HMF in [BMim]Cl. 

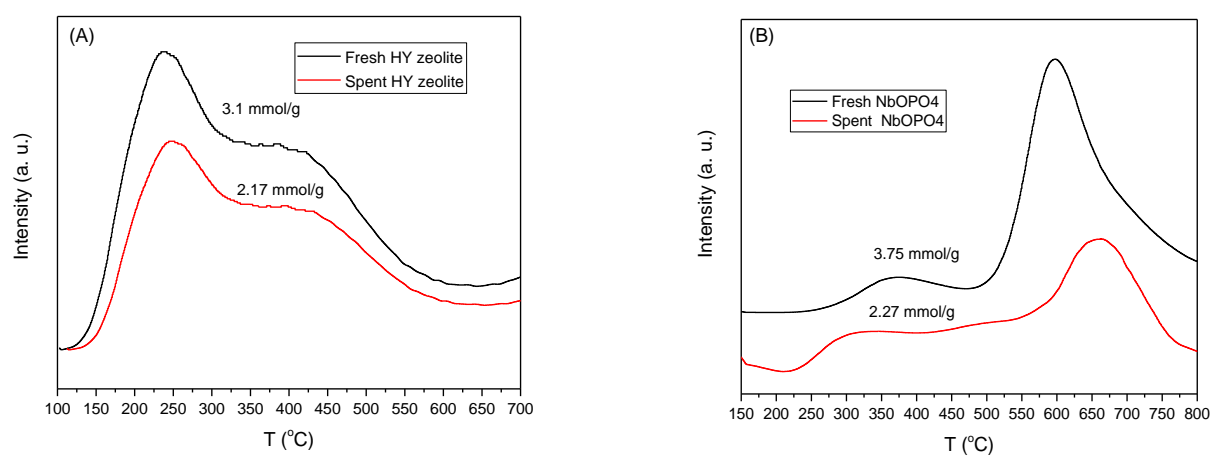

Figure S6. $\mathrm{NH}_{3}$-TPD profiles obtained on (A) HY zeolite and (B) $\mathrm{NbOPO}_{4}$.
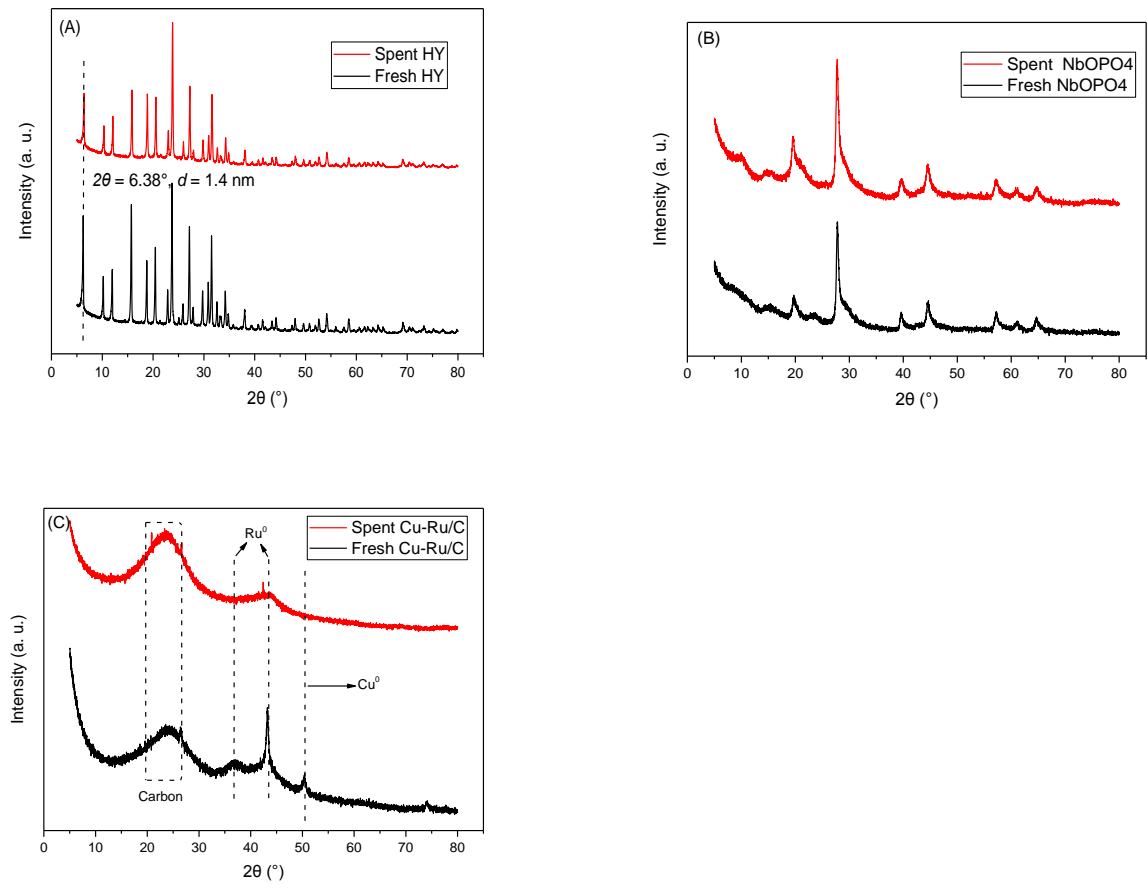

Figure S7. XRD patterns for the fresh and spent catalyst of (A) HY zeolite, (B) $\mathrm{NbOPO}_{4}$, and (C) $\mathrm{Cu}-\mathrm{Ru} / \mathrm{C}$. 

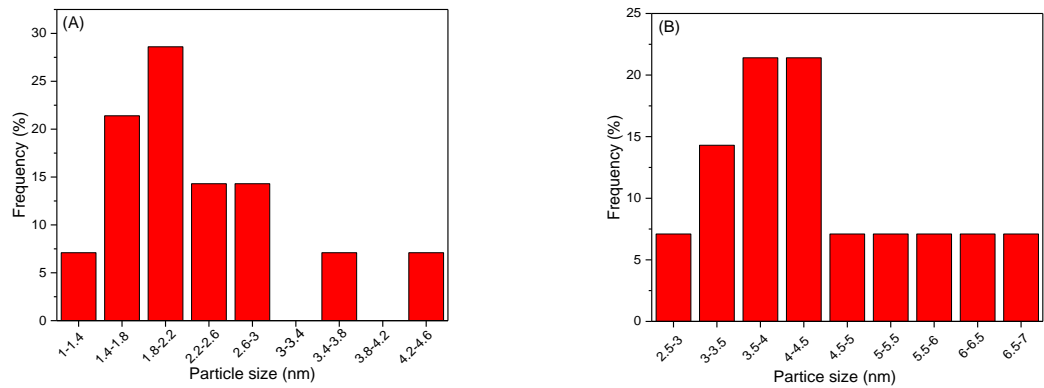

Figure S8. Average particle size distribution of $\mathrm{Cu}$, $\mathrm{Ru}$ over $(\mathrm{A})$ fresh $\mathrm{Cu}-\mathrm{Ru} / \mathrm{C}$ and $(\mathrm{B})$ spent $\mathrm{Cu}-\mathrm{Ru} / \mathrm{C}$.

\section{REFERENCES}

[1] Guo, J.; Zhang, X., Metal-ion interactions with sugars. The crystal structure and FTIR study of an $\mathrm{SrCl}_{2}$-fructose complex. Carbohyd. Res. 2004, 339 (8), 1421-1426. [2] Feng, W. Q.; Lu, Y. H.; Ying, C.; Lu, Y. W.; Tao, Y., Thermal stability of imidazolium-based ionic liquids investigated by TG and FTIR techniques. J. Therm. Anal. Calorim. 2016, 125 (1), 143-154. 